\title{
Advection and dispersal of small magnetic elements in the very quiet Sun
}

\author{
R. Manso Sainz ${ }^{1,2}$, M. J. Martínez González ${ }^{1,2}$, and A. Asensio Ramos ${ }^{1,2}$ \\ ${ }^{1}$ Instituto de Astrofísica de Canarias, vía Láctea s/n, 38205 La Laguna, Tenerife, Spain \\ e-mail: rsainz@iac.es \\ 2 Departamento de Astrofísica, Universidad de La Laguna, 38205 La Laguna, Tenerife, Spain
}

Received 6 April 2011 / Accepted 1 June 2011

\begin{abstract}
We track small magnetic structures on very quiet regions (internetwork) of the Sun. We follow the footpoints of small-scale magnetic loops that appear on the photosphere at granular scales using spectropolarimetric and magnetographic data obtained with Hinode. We find two different regimes for their wanderings. Within granules (where they appear), they seem to be passively advected by the plasma - which is justified by their relatively low magnetic flux $\left(\sim 10^{16} \mathrm{Mx}\right)$, and magnetic field strength $(\sim 200 \mathrm{G})$. The plasma flow thus traced is roughly laminar with a characteristic mean velocity of $2 \mathrm{~km} \mathrm{~s}^{-1}$ and very low vorticity. Once the magnetic markers reach intergranular lanes, they remain there and are buffeted by the random flows of neighbouring granules and turbulent intergranules, follow random walks, and disperse across the solar surface with a diffusion constant of $195 \mathrm{~km}^{2} \mathrm{~s}^{-1}$. While on their intergranular random walking, they may fall close to whirlpools (on scales $\lesssim 400 \mathrm{~km}$ ) associated with convective downdrafts, similar to the events recently reported in mesogranular and supergranular cell boundaries tracking magnetic bright points, which provides additional evidence that these events are ubiquitous on the solar surface.
\end{abstract}

Key words. Sun: atmosphere - Sun: granulation - convection - Sun: surface magnetism

Tracking small-scale magnetic elements in the solar surface is an elementary but important technique for understanding the intrinsic dynamics of small-scale magnetic fields and to characterize their topological evolution (e.g., Berger \& Title 1996; Welsch \& Longcope 2003; Rouppe van der Voort et al. 2005). It is important in connection with the coronal heating problem (e.g., through the link between photospheric plasma motions and the braiding and knotting of magnetic field lines in upper layers; van Ballegooijen et al. 1998), the global dynamo and solar cycle (how fields disperse on the solar surface; e.g., Berger et al. 1998), and also to trace horizontal flows (e.g., Nisenson et al. 2003; Bonet et al. 2008). Most works trace the paths of G-band bright points, which are thought to be related to strongly magnetized structures (Spruit 1977; Steiner et al. 2001). Otherwise, magnetograms with relatively low spatial resolution and sensitivity are used, which mostly reveal network regions as well - the strongly magnetized boundaries of supergranulation cells. Only sensitive spectropolarimetry has made it possible to access directly the weaker magnetism inside this cellular pattern - the so-called internetwork (Martínez González et al. 2007; de Wijn et al. 2008). This has led to the discovery that these fields may also organize as structures at and below granular scales, and that they are dynamic and long-lived. The rising hot plasma brings a well organized magnetic field from below the surface which, as the plasma moves towards the granular boundary radiating and cooling, develops into the general shape of an $\Omega$-loop (Martínez González et al. 2007). The crown of the loop, where the magnetic field is horizontal, is the first to appear, and then vanishes after crossing the line formation layer. The footpoints, on the contrary, where the magnetic field is mostly vertical, remain visible at all times once they appear (Centeno et al. 2007; Martínez González \& Bellot Rubio 2009).

The footpoints of small magnetic loops are particularly interesting for tracing photospheric flows because of their low magnetic flux and small spatial scale. The magnetic flux in small magnetic loops is $\sim 10^{16}-10^{17} \mathrm{Mx}$, and the intrinsic magnetic field strength is $200 \mathrm{G}$ (Martínez González et al. 2010); bright points, on the contrary, are thought to have field strengths of the order of $\sim \mathrm{kG}$ (Spruit 1977). Additionally, because of the small scale of these events, we can aim at tracing intragranular flows bright points are confined to intergranular lanes. But it is also interesting to consider how the combined flux of these structures, which are present at all times during the solar cycle, disperse all over the solar surface.

In this note we report on the wanderings of the footpoints of these small-scale loops that appear in the very quiet (internetwork) Sun. First, as they are passively advected by the plasma flow and then, as they disperse away contributing to the global diffusion of magnetic flux through the solar surface.

We analyse here the observations reported by Martínez González \& Bellot Rubio (2009). They comprise seven series of scans of the quiet Sun, covering a field of view (FOV) of $2^{\prime \prime} 7 \times 400^{\prime} 6$, with a time cadence of $28 \mathrm{~s}$ for 2-6 h, performed with the spectro-polarimeter (SP; Lites et al. 2001), of the Solar Optical Telescope (SOT) onboard Hinode (Kosugi et al. 2007), which records the Fe I $630 \mathrm{~nm}$ doublet. Additionally, we consider magnetograms taken with the Hinode Narrowband Filter Imager (NFI; Tsuneta et al. 2008), at $\pm 11.5 \mathrm{pm}$ from the line core of the $\mathrm{Mg}$ I $517.3 \mathrm{~nm}$ line, covering a wider FOV of $15^{\prime \prime} .4 \times 65^{\prime \prime} .3$. 


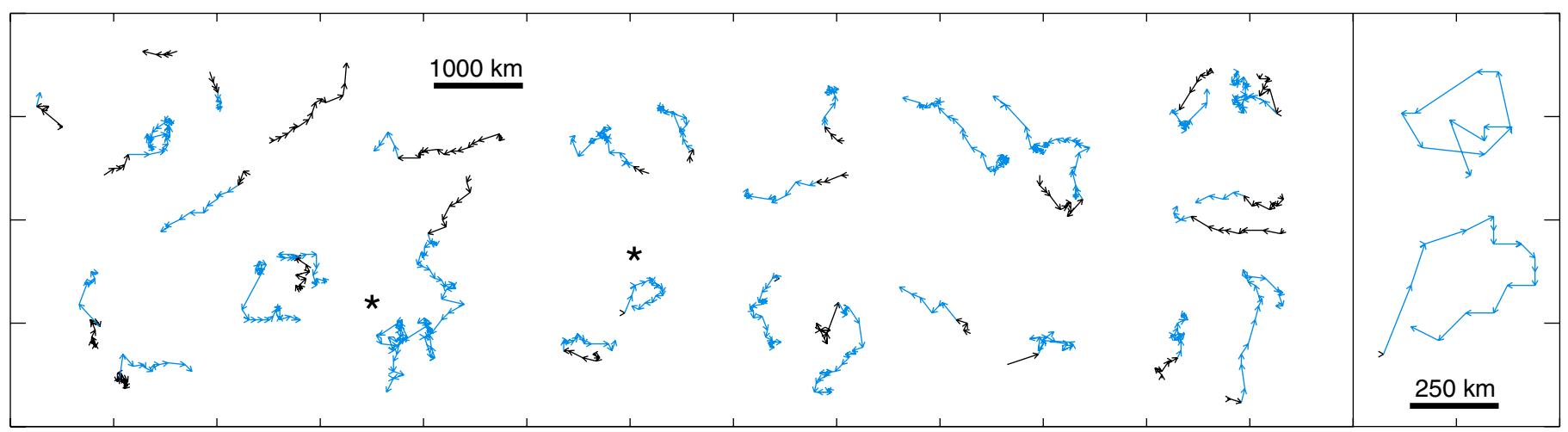

Fig. 1. Sample trajectories followed by the footpoints of small-scale magnetic loops on the quiet Sun. Black arrows refer to the part of the trail over granules, blue (grey) arrows indicate the path covered on intergranular lanes. On the right, enlarged image of the two intergranular paths marked with a star.

The FOV covered by the spectropolarimetric scans is relatively narrow and the evolution of the loops is soon missed. Fortunately, these structures raise to the upper photosphere and may even reach the chromosphere (Martínez González \& Bellot Rubio 2009). Approximately $5 \mathrm{~min}$ after appearing on the surface, the counterpart on the upper photosphere of the footpoints can be seen cospatially on the Mg I magnetogram. This allows us to follow the footpoints throughout the wider area covered by the NFI instrument.

The small loop footpoints are detected and tracked manually. They appear in pairs with opposite polarities at the two ends of a linear polarization region, and they can be tracked and followed at successive time frames even after the linear polarization is no longer detected. The extension of a footpoint on the magnetogram depends on the detection threshold. Typically, we used three times the noise level over the background, which gives areas of $\sim 0.46 \operatorname{arcsec}^{2}$. We define the "position" of the footpoint as the barycenter of this area in the magnetograph. Figure 1 shows samples of footpoint paths.

Loops, and hence, their footpoints, appear inside granules. We followed them until they reached an intergranular lane, where they remain until they either disappear from view or cannot be unambiguously traced anymore (e.g., because they apparently merge with other structures). Both stages are shown in Fig. 1.

For a given path $X(t), Y(t)$, we compute the first differences $\Delta X(t)=X(t)-X(t-T), \Delta Y(t)=Y(t)-Y(t-T)(T=28 \mathrm{~s}$ is the temporal cadence of the observations), and from these, the variances $\left\langle\Delta^{2} X\right\rangle,\left\langle\Delta^{2} Y\right\rangle$, and correlation $\langle\Delta X \Delta Y\rangle$, where the averages are taken for the whole path length. We define the polarization of the path as $p=1-4\left(\left\langle\Delta^{2} X\right\rangle\left\langle\Delta^{2} Y\right\rangle-\langle\Delta X \Delta Y\rangle^{2}\right) /\left(\left\langle\Delta^{2} X\right\rangle+\left\langle\Delta^{2} Y\right\rangle\right)^{2}$. This quantity is invariant with respect to changes of the axes $X$ and $Y$. For a random path, $\left\langle\Delta^{2} X\right\rangle=\left\langle\Delta^{2} Y\right\rangle$ while $\langle\Delta X \Delta Y\rangle^{2}=0$; hence, $p=0^{1}$. For the extreme case of a completely directed trajectory $(Y(t) \propto X(t)), p=1$. For a random walk with drift, $p$ varies between both extremes, depending on the respective weight of random walk and drift.

\footnotetext{
1 This is a necessary, but not sufficient condition: non-random paths with $p=0$ may exist. A complete statistical description of the path would require us to consider additional second order correlations $(\langle\Delta X(t) X(t+\tau)\rangle,\langle\Delta X(t) Y(t+\tau)\rangle,\langle\Delta X(t) Y(t+\tau)\rangle)$ and a more general expression for the polarization. This is beyond the scope of this note. Our aim here is just to manifest quantitatively the different behaviours between granular and intergranular paths (see below).
}

Figure 2 shows the distribution of step lengths $\left(\sqrt{\Delta^{2} X+\Delta^{2} Y}\right)$ in granular (panel a) and intergranular (panel b) paths. A Rayleigh distribution (corresponding to a Gaussian distribution for $\Delta X(t)$ and $\Delta Y(t))$ with $\sigma=65 \mathrm{~km}$ fits both distributions. Panels $\mathrm{c}$ and d show the distribution of polarization for paths with at least 6 steps on a granule, or at least 12 on the intergranules, respectively. Solid lines show the expected distribution calculated from 500000 realizations of random paths (with and without drift) with the same number of steps as in the observed walkings and step lengths taken from the distribution in panels a and $b$. The granular distribution is not compatible with a pure random walk. At least a drift $\sim 4 \mathrm{~km} \mathrm{~s}^{-1}$ is required for some walkers; for others, the drift completely dominates over any random meandering $(p \approx 1)$. By contrast, the intergranular behaviour is compatible with a pure random walk without drift.

Figure 2e shows the end-to-end square distance $D^{2}=[X(t)-$ $X(0)]^{2}+[Y(t)-Y(0)]^{2}$ covered by 46 markers (both footpoints of 23 small-scale loop events) from the position where they appear for the first time on a granule, until they reach an intergranular lane. We did not follow the evolution beyond $5 \mathrm{~min}$ because very few of them remain longer on the granule and their statistical significance is low.

The trajectories of individual walkers may show large fluctuations as Figs. 2e-f more clearly shows (grey lines). Statistically, the behaviour of the walkers is characterized by the scaling of the mean-square distance covered with time: $\left\langle D^{2}\right\rangle \propto t^{a}$, which can be diffusive ( $a=1)$, subdiffusive $(a<1)$, superdiffusive ( $a>1)$, or ballistic $(a=2)$.

A fit on a log-log plot shows a superdiffusive behaviour $\left\langle D^{2}\right\rangle \propto t^{1.7}$ within the granules. At the spatial and temporal scales we are considering, the trajectories of the magnetic elements over granules exibit a behaviour typical of passive corks over a laminar flow - relatively directed trajectories $(p \gtrsim 0.2)$, and superdiffusion -, rather than turbulence - i.e., chaotic, unpolarized, diffusive trajectories. A linear fit to $\langle D\rangle$ versus time gives a mean velocity of the horizontal flows of $2 \mathrm{~km} \mathrm{~s}^{-1}$. Their behaviour can be interpreted with a simple model of a random walk with drift, though in some instances the trajectory is purely ballistic. The random walk component of the magnetic element path tracks the "turbulent" component of the granular flow; the drift, the "gentle laminar flow" (Stein \& Nordlund 1998) to the intergranular lane.

Consistent with the laminar character of the granular flow, we find a relatively low estimate for the vorticity on the 

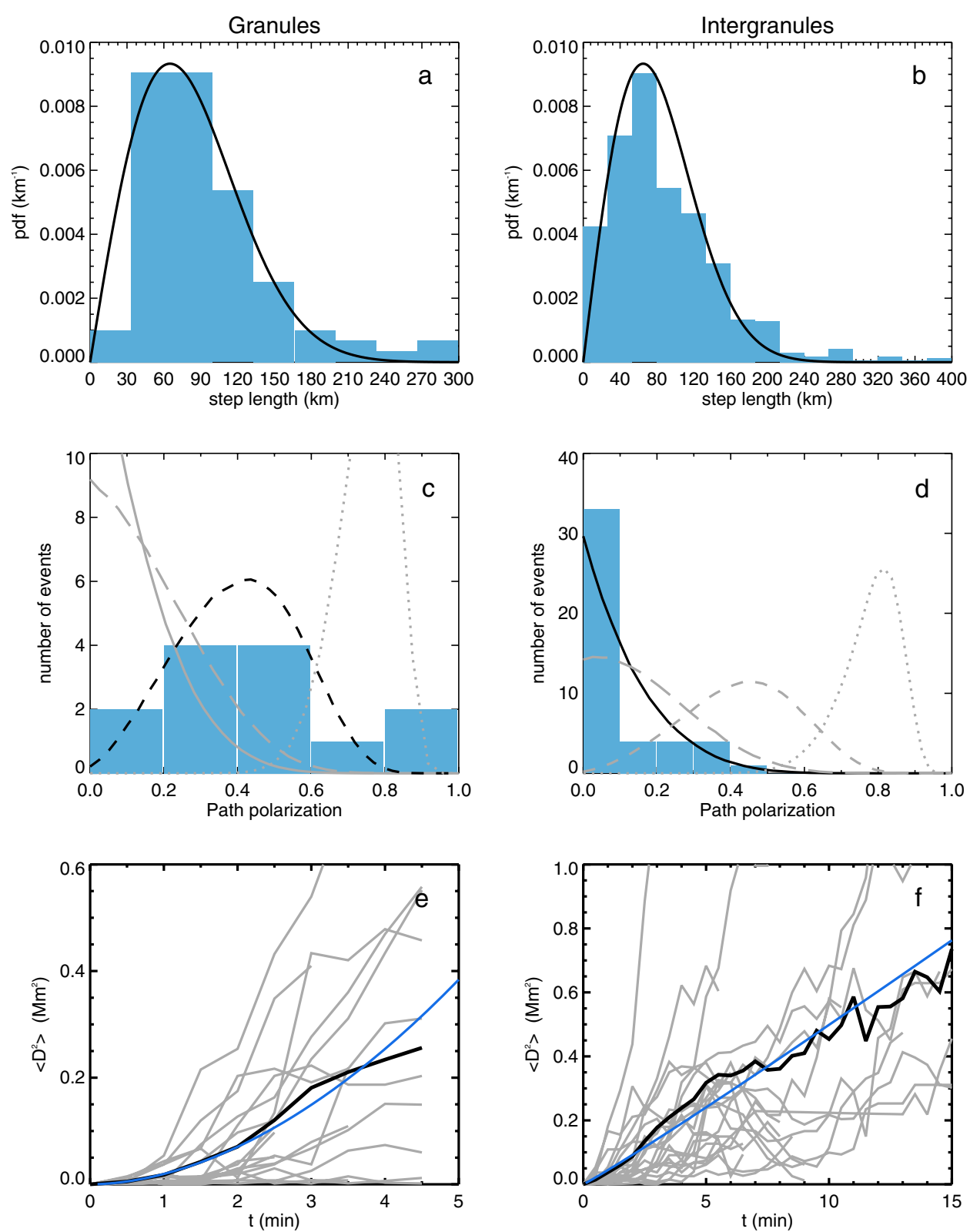

Fig. 2. Statistical properties of the walks followed by footpoints while in granules (left panels), and in intergranular lanes (right panels). a), b) distribution (probability density function, pdf) of the observed step lengths (histogram), and fit to a Rayleigh distribution $p d f(s)=\frac{s}{\sigma^{2}} \exp \left(-s^{2} / 2 \sigma^{2}\right.$ ) with $\sigma=65 \mathrm{~km}$ (solid line). c) distribution of path polarization (see text) for paths with at least 6 steps (3 min) on a granule. Solid line shows expected distribution calculated from 6-step random paths assuming the step distribution of panel a); long-dashed, dashed, and dotted lines, the expected distribution when drifts of 2.4, 4.6 , and $9.7 \mathrm{~km} \mathrm{~s}^{-1}$, respectively, is added. A reasonable fit is achieved for the black line. d) as in panel c) but for intergranular paths with at least 12 steps (average of 24 steps). Best fit (black line) for pure random walk without drift. e), f) end-to-end distance squared $D^{2}=\left[X(t)-X\left(t_{0}\right)\right]^{2}+\left[Y(t)-Y\left(t_{0}\right)\right]^{2}\left(t_{0}=0\right.$, for granules in panel e), or the moment when the intergranular path begins in panel f), covered by magnetic elements, and mean square distance $\left\langle D^{2}\right\rangle$ averaged over realizations (thick black line). Blue (dark grey) lines show that best fits to $\left\langle D^{2}\right\rangle \sim t^{a}$ are obtained with $a=1.7$ and $a=0.96$ (diffusive regime), for granules and intergranules, respectively.

granular flow. At the beginning of their wandering, the loop footpoints are just $\sim 500 \mathrm{~km}$ apart. The vertical component of the vorticity $\omega_{z}=\partial_{x} v_{y}-\partial_{y} v_{x}\left(v_{x}\right.$ and $v_{y}$ being the horizontal components of the velocity), can be estimated as $\omega_{z} \approx\left[\Delta Y(t)^{+}-\right.$ $\left.\Delta Y(t)^{-}\right] /\left(T \Delta_{x}\right)-\left[\Delta X(t)^{+}-\Delta X(t)^{-}\right] /\left(T \Delta_{y}\right)$, where + and - stand for both footpoints at the moment $t$ of their closest encounter (i.e., at $t=0$ ), when $\Delta_{x}=X(t)^{+}-X(t)^{-}$and $\Delta_{y}=Y(t)^{+}-Y(t)^{-}$. Averaging over 15 loops, $\left\langle\omega_{z}^{2}\right\rangle^{1 / 2} \approx 0.017 \mathrm{~s}^{-1}$.

Laminar behaviour in the granular flow has been anticipated by numerical simulations (e.g., Stein \& Nordlund 1998; Nordlund et al. 2009). The rise of the hotter plasma in the convective cell (granule) in a strongly stratified atmosphere (low density scale height) leads to strongly divergent horizontal flows just due to mass conservation. Simulations by Stein \& Nordlund (1998; see their Fig. 18) show low vorticities $\omega \leq 0.05 \mathrm{~s}^{-1}$ when a parcel of plasma is crossing the granule, in agreement with our estimate.

Once they reach an intergranular lane, the footpoints' wandering changes regime and becomes close to a random walk $\left(\left\langle D^{2}\right\rangle \propto t^{0.96}\right)$. Figure $2 \mathrm{f}$ shows the quadratic distance $D^{2}$ covered by 46 individual footpoints while on intergranular lanes. The ensemble average of random walks quickly converges to a well-defined behaviour for $\left\langle D^{2}\right\rangle$. We calculate this average when at least 6 random walks are available at a given time (this is 
always the case for $t \leq 15 \mathrm{~min}$ ). In just five cases the trajectories can be traced beyond $15 \mathrm{~min}$, but these last time steps are not considered statistically meaningful for averaging. From a linear regression we obtained a slope of $777 \mathrm{~km}^{2} \mathrm{~s}^{-1}$, which implies a diffusion coefficient (diffusivity) in two dimensions $K \equiv\left\langle D^{2}\right\rangle / 4 t \approx 195 \mathrm{~km}^{2} \mathrm{~s}^{-1}$.

While on their intergranular random walk, we discovered two instances in which the magnetic elements describe a vortical motion (see the paths marked with a star in Fig. 1). They spiral at scales $\lesssim 400 \mathrm{~km}$. One structure describes a full circle in $7 \mathrm{~min}$ before disappearing below the detection limit; the other follows a downwards spiral for $5 \mathrm{~min}$ and then continues on its way. These events are very similar to the whirlpools observed by Bonet et al. (2008) when tracking magnetic bright points (BPs) in moderately magnetized (network) areas of the Sun that are engulfed by downdrafts. Because the BPs are associated to relatively strong concentrations of magnetic flux, whirlpools traced through BPs seem to trace supergranulation - in turn, associated to the network. Our spectropolarimetric detection here of similar whirlpools (see also, Balmaceda et al. 2010) in very quiet internetwork regions in a relatively limited FOV and time span additionally supports the impression that these events are, in fact, ubiquitous on the solar surface.

The diffusion coefficient that quantifies the dispersal of magnetic elements through the solar surface has been measured with different techniques at different activity regions on the Sun. Mosher (1997) derived $200-400 \mathrm{~km}^{2} \mathrm{~s}^{-1}$. Applying auto- and cross-correlation to videomagnetograms, Wang (1988) reports $\sim 150 \mathrm{~km}^{2} \mathrm{~s}^{-1}$ for network regions. Tracking magnetic fluxtubes around an active region, Schrijver \& Martin (1990) give $110 \mathrm{~km}^{2} \mathrm{~s}^{-1}$ at the core of a plage region and $250 \mathrm{~km}^{2} \mathrm{~s}^{-1}$ in the surrounding quieter areas. Komm et al. (1995) apply crosscorrelation techniques to high-resolution magnetograms and get 120-230 km $\mathrm{km}^{-1}$. Berger et al. (1998) track magnetic bright points in the G-band and obtain $50-80 \mathrm{~km}^{2} \mathrm{~s}^{-1}$, while artificial passive corks on the velocity field reconstructed with local correlation gives 77,176 , and $285 \mathrm{~km}^{2} \mathrm{~s}^{-1}$, for magnetic, network, and quiet regions respectively. Hagenaar et al. (1999) find different diffusivities for short time-scales (70-90 $\mathrm{km}^{2} \mathrm{~s}^{-1}$ below $3 \mathrm{~h}$ ) and long time-scales (200-250 $\mathrm{km}^{2} \mathrm{~s}^{-1}$ above $8 \mathrm{~h}$ ) by tracking magnetic concentrations with fluxes $10^{18}-10^{19} \mathrm{Mx}$.

These works show some evidence that the stronger magnetized elements diffuse more slowly than less magnetized ones. Nonetheless, the diffusivity in all cases falls short of the $\sim 600 \mathrm{~km}^{2} \mathrm{~s}^{-1}$ required by semiempirical models to reproduce the migration of magnetic flux towards the poles and the polarity inversion with an 11 year cycle (Sheeley 2005) within the Babcock-Leighton model (Leighton 1964; Babcock 1961). Schrijver et al. (1996) argue that there is, indeed, an observational bias towards the strongest magnetized, hence slower, magnetic features. Accounting for the less magnetized, faster magnetic elements, the diffusion coefficient for the dispersal of photospheric magnetic flux would be according to theoretical expectations $\left(\sim 600 \mathrm{~km}^{2} \mathrm{~s}^{-1}\right)$. Our findings reported here, however, argue against this. One possible way to reconcile this would be the presence of horizontal flows leading diffusion at different spatial scales. What we measure here would be diffusion at the lowest (granular) scale. This would rest on top of diffusive flows at larger scales (e.g., supergranular scales), and the total diffusivity would be the result of adding up all of them at all scales.
Tracking small-scale low-flux magnetic elements allows tracing horizontal flows across the very quiet Sun at the lowest spatial scales - even at intragranular scales. These patches last for a few minutes before diffusing and falling below the detection limit, or merging with nearby low-flux spots and loosing their identity. This makes it difficult to follow the dispersal of magnetic flux on long timescales. On short timescales ( $\$ 20 \mathrm{~min}$ ), however, they trace the small-scale intragranular flows and resolve the vortical flows on intergranular lanes. There is evidence for a disorganised, "turbulent" component in the intragranular flow that must be observed with a time cadence better than $30 \mathrm{~s}$ to be resolved.

This work should be extended by considering a more complete statistical sample. This requires polarimetric observations covering a larger area of the Sun with a relatively good time cadence. A good candidate for this would be, for example, the IMaX magnetometer (Martínez Pillet et al. 2011) onboard the Sunrise balloon (Solanki et al. 2010; Barthol et al. 2011). Additionally, the number of magnetic elements mapping the flow can be increased by using isolated markers not necessarily associated to the footpoints of small-scale loops.

Acknowledgements. We are very grateful to M. Collados for fruitful discussions that led to improvements on the letter. Finantial support by the Spanish Ministry of Science through projects AYA2010-18029 (Solar Magnetism and Astrophysical Spectropolarimetry) and CONSOLIDER INGENIO CSD200900038 (Molecular Astrophysics: The Herschel and Alma Era) is gratefully acknowledged.

\section{References}

Babcock, H. W. 1961, ApJ, 133, 572

Balmaceda, L., Vargas Domínguez, S., Palacios, J., Cabello, I., \& Domingo, V. 2010, A\&A, 513, L6

van Ballegooijen, A. A., Nisenson, P., Noyes, R. W., et al. 1998, ApJ, 509, 435

Barthol, P., Gandorfer, A., Solanki, S. K., et al. 2011, Sol. Phys., 268, 1

Berger, T. E., \& Title, A. M. 1996, ApJ, 463, 365

Berger, T. E., Löfdahl, M. G., Shine, R. A., \& Title, A. M. 1998, ApJ, 506, 439

Bonet, J. A., Márquez, I., Sánchez Almeida, J., Cabello, I., \& Domingo, V. 2008, ApJ, 687, L131

Centeno, R., Socas-Navarro, H., Lites, B., et al. 2007, ApJ, 666, L137

Hagenaar, H. J., Schrijver, C. J., Title, A. M., \& Shine, R. A. 1999, ApJ, 511, 932

Komm, R. W., Howard, R. F., \& Harvey, J. W. 1995, Sol. Phys., 158, 213

Kosugi, T., Matsuzaki, K., Sakao, T., et al. 2007, Sol. Phys., 243, 3

Leighton, R. B. 1964, ApJ, 140, 1547

Martínez González, M. J., \& Bellot Rubio, L. R. 2009, ApJ, 700, 1391

Martínez González, M. J., Collados, M., Ruiz Cobo, B., \& Solanki, S. K. 2007, A\&A, 469, L39

Martínez González, M. J., Manso Sainz, R., Asensio Ramos, A., \& Bellot Rubio, L. 2010, ApJ, 714, L94

Martínez Pillet, V., et al. 2010, Sol. Phys., 268, 57

Mosher, J. M. 1977, Ph.D. thesis, California Inst. Technol.

Nisenson, P., van Ballegooijen, A. A., de Wijn, A. G., \& Sütterlin, P. 2003, ApJ, 587,458

Nordlund, Å, Stein, R. F., \& Asplund, M. 2009, Living Rev. Sol. Phys., 6, 2

Rouppe van der Voort, L. H. M., Hansteen, V. H., Carlsson, M., et al. 2005. A\&A, 435, 327

Schrijver, C. J., \& Martin, S. F. 1990, Sol. Phys., 129, 95

Sheeley, N. R., Jr. 2005, Living Rev. Sol. Phys., 2, 5

Solanki, S. K., Barthol, P., Danilovic, S., et al. 2010, ApJ, 723, L127

Spruit, H. C. 1977, Sol. Phys., 55, 3

Stein, R. F., \& Nordlund, Å 1998, ApJ, 499, 914

Steiner, O., Hauschildt, P. H., \& Bruls, J. 2001, A\&A, 372, L13

Tsuneta, S., Ichimoto, K., Katsukawa, Y., et al. 2008, Sol. Phys., 249, 167

Wang, H. 1988, Sol. Phys., 116, 1

Welsch, B. T., \& Longcope, D. W. 2003, ApJ, 588, 620

de Wijn, A. G., Lites, B. W., Berger, T. E., et al. 2008, ApJ, 684, 1469 